\title{
Identifiability analysis of mathematical models of immunology and epidemiology
}

\author{
V. Latyshenko ${ }^{1,2 *}$, O. Krivorotko ${ }^{1,2}$, S. Kabanikhin ${ }^{1}$ \\ ${ }^{1}$ Institute of Computational Mathematics and Mathematical Geophysics SB RAS, Novosibirsk, Russia \\ ${ }^{2}$ Novosibirsk State University, Novosibirsk, Russia \\ *e-mail: Latushenko_varia@mail.ru
}

Key words: ordinary differential equations, inverse problems, practical identifiability, sensitivity-based analysis, immunology, epidemiology

Motivation and Aim: Lately ordinary differential equations (ODEs) became the predominant tool in the field of biology (immunology, epidemiology), medicine (pharmacokinetics, tomography), sociology, economics, etc. Mathematical models are based on systems of ODEs (regular, nonlinear) and their coefficients characterize, for example, individual characteristics of patient and population in epidemiology field and should be identify for construction of individual treatment plan and the best forecasting of epidemic.

Methods and Algorithms: Before determining unknown parameters of models (inverse problem [1]), we should understand: whether is there a solution? how many parameters can we determine from the available data? how many measurements (additional information about solution of ODEs in fixed times) need to be taken to determine the required set of parameters? These questions are answered by analysis of the identifiability [2]. The identifiability is the ability to uniquely determine the values of parameters with sufficient data volume. Analysis of the identifiability represents a study for a deep understanding of the model.

In this work we analyze several methods of identifiability analysis. For practical identifiability we use methods of correlation matrix and Monte Carlo simulation, for sensitivity-based analysis we use orthogonal and eigenvalues methods.

Results: Methods of identifiability was applied to different mathematical models of biology (model of dynamic HIV-infection [3], model of spread of co-infection HIV and tuberculosis [4]). Sequences of identified parameters were obtained for each mathematical model.

Acknowledgements: The authors were supported by the grant No. AP05134121 of the Ministry of Education and Science of Republic of Kazakhstan, Ministry of Education and Science of Russian Federation and by the Scholarship of the President of RF No. MK-1214.2017.1. and the grant No. 18-71-10044 of Russian Scientific Found (RScF).

\section{References}

1. Kabanikhin S.I. (2008) Definitions and examples of inverse and ill-posed problems. Inverse Ill-posed Probl. 16:317-357

2. Miao H., Xia X., Perelson A.S., We H. (2011) On identifiability of nonlinear ODE models and applications in viral dynamics. SIAM Rev. Soc. Ind. Appl. Math. 53(1):3-39.

3. Adams B.M., Banks H.T. et al. (2005) HIV dynamics: Modeling, data analysis, and optimal treatment protocols. Journal of Computational and Applied Mathematics. 184:10-49.

4. Roeger L.W., Feng Z., Castillo-Chavez C. (2009) Modelling TB and HIV Coinfections. Mathematical biosciences and engineering. 6(4):815-837. 